\title{
Is Physical Activity Associated with Less Depression and Anxiety During the COVID-19 Pandemic? A Rapid Systematic Review
}

\author{
Sebastian Wolf ${ }^{1,2}\left(\right.$ Britta Seiffer $^{1,2} \cdot$ Johanna-Marie Zeibig $^{1,2} \cdot$ Jana Welkerling $^{1,2} \cdot$ Luisa Brokmeier $^{3}$. \\ Beatrice Atrott ${ }^{1,2} \cdot$ Thomas Ehring $^{4} \cdot$ Felipe Barreto Schuch $^{5}$
}

Accepted: 1 April 2021 / Published online: 22 April 2021

(c) The Author(s) 2021

\begin{abstract}
Background The Covid-19 pandemic is affecting the entire world population. During the first spread, most governments have implemented quarantine and strict social distancing procedures. Similar measures during recent pandemics resulted in an increase in post-traumatic stress, anxiety and depression symptoms. The development of novel interventions to mitigate the mental health burden are of utmost importance.

Objective In this rapid review, we aimed to provide a systematic overview of the literature with regard to associations between physical activity (PA) and depression and anxiety during the COVID-19 pandemic.

Data Source We searched major databases (PubMed, EMBASE, SPORTDiscus, and Web of Science) and preprint servers (MedRxiv, SportRxiv, ResearchGate, and Google Scholar), for relevant papers up to 25/07/2020.

Study Eligibility Criteria We included observational studies with cross-sectional and longitudinal designs. To qualify for inclusion in the review, studies must have tested the association of PA with depression or anxiety, using linear or logistic regressions. Depression and anxiety must have been assessed using validated rating scales.

Study Appraisal and Synthesis Methods Effect sizes were represented by fully adjusted standardized betas and odds ratios (OR) alongside 95\% confidence intervals (CI). In case standardized effects could not be obtained, unstandardized effects were presented and indicated.

Results We identified a total of 21 observational studies (4 longitudinal, 1 cross-sectional with retrospective analysis, and 16 cross-sectional), including information of 42,293 (age 6-70 years, median female $=68 \%$ ) participants from five continents. The early evidence suggests that people who performed PA on a regular basis with higher volume and frequency and kept the PA routines stable, showed less symptoms of depression and anxiety. For instance, those reporting a higher total time spent in moderate to vigorous PA had 12-32\% lower chances of presenting depressive symptoms and $15-34 \%$ of presenting anxiety. Conclusion Performing PA during Covid-19 is associated with less depression and anxiety. To maintain PA routines during Covid-19, specific volitional and motivational skills might be paramount to overcome Covid-19 specific barriers. Particularly, web-based technologies could be an accessible way to increase motivation and volition for PA and maintain daily PA routines.
\end{abstract}

Sebastian Wolf

sebastian.wolf@uni-tuebingen.de

1 Department of Psychology, Institute of Clinical Psychology and Psychotherapy, University of Tuebingen, Tuebingen, Germany

2 Institute of Sport Science, Department of Education \& Health Research, University of Tuebingen, Tuebingen, Germany

3 Mannheim Institute of Public Health, Mannheim Medical Faculty, University of Heidelberg, Mannheim, Germany

4 Department of Psychology, LMU Munich, Munich, Germany

5 Department of Sports Methods and Techniques, Federal University of Santa Maria, Santa Maria, Brazil 


\section{Key Points}

The Covid-19 pandemic increased symptoms of anxiety and depression symptoms. Those reporting a higher total time spent in moderate to vigorous physical activity, had $12-32 \%$ lower chances of presenting depressive symptoms and $15-34 \%$ of presenting anxiety.

The promotion of physical activity habits and routines might be a cost-effective and comprehensive worldwide applicable strategy to overcome the severe gap between people in need and people receiving mental health care, especially in low-income countries with even non-existing mental health supplies.

Web-based technologies might be promising tools to increase motivation and volition for PA and maintain daily physical activity routines even under pandemicspecific barriers. However, there is a clear need for more systematic research for effectively and safely usable apps or web-based programs to prevent psychiatric disorders through physical activity.

\section{Introduction}

With 106,125,682 confirmed cases all over the world (up to February 10th, 2021), COVID-19 is a global public health emergency. COVID-19 is characterized by a fast human-tohuman transmission through droplet or close contact. Given the lack of appropriate treatments and vaccines during the early stage of the pandemic, many countries implemented procedures recommended by the World Health Organization (WHO) [1], such as the isolation of symptomatic patients, quarantining individuals with the history of contact with COVID-19 infected persons, and further anti-contagion policies such as mandatory stay at home or business closures. Those anti-contagion policies substantially reduced exponential growth rates $[2,3]$.

Quarantine and social distancing measures had already been successfully enforced during earlier pandemics, such as the 2003 outbreak of SARS and the 2014 outbreak of Ebola [4]. However, studies on the effects of these measures have reported elevated symptoms of anxiety, post-traumatic stress, and depressive disorders, as well as a $30 \%$ higher suicide rates in populations impacted by these measures $[5$, 6]. These findings are being replicated during the Covid-19 pandemic with multiple studies reporting elevated prevalences of depression and anxiety [7-11].
Notably psychiatric disorders result in a considerable burden of disease, accounting for $6.7 \%$ of overall disability-adjusted life years [12] and being attributable to $14.3 \%$ of death worldwide [13]. Despite the high burden of psychiatric disorders, there is a severe gap between people in need and people receiving mental health care [14]. This general treatment gap is especially severe in low- and middle-income countries, where $76-85 \%$ of people with mental disorders do not receive any treatment [15]. The latest WHO "mental health Atlas" indicates that only 95.6 out of 100000 depressed cases worldwide receive any professional mental health care, whereas the treatment prevalence in high-income countries is 16-times higher compared to low-income countries [16]. Although there is no current global data available, the treatment gap is assumed to be much higher during or after the Covid-19 pandemic. Access to general mental health care might be restricted for several reasons, including supply priorities that being focused on Covid-19 infections, medication shortages, prohibition of face-to-face psychotherapeutic sessions of psychological treatment, closing of inpatient facilities to mention only some reasons. Indeed, current international position papers point out a clear need to adapt and improve mental health services worldwide due to these specific challenges during and after the pandemic $[17,18]$. To mitigate the negative mental health consequences of pandemics, evidence suggests that policymakers should ensure quarantine measures to be as short as possible, to provide adequate general supplies for basic needs, give people as much information as possible and strengthen social support and communication among people affected by the pandemic [4]. A recently published position paper on research priorities for mental health science regarding COVID-19 [18] demands the interdisciplinary development of novel interventions to protect mental wellbeing by mechanistically based approaches to strengthen altruism and prosocial behavior. Among others, physical activity (PA) interventions are highlighted as a promising approach. PA is defined as any bodily movement produced by skeletal muscles that results in energy expenditure and exercise is defined as PA, which is planned, structured, and repetitive, with the primary aim to improve or maintain physical fitness [19]. International PA guidelines recommend $150 \mathrm{~min}$ of moderate or $75 \mathrm{~min}$ of vigorous intensity PA per week for optimal physical and mental health benefits [20]. Indeed, in pre-pandemic times PA has been identified as a protective factor against incident depression [21] and anxiety [22]. However, decreased levels of PA were observed in the general population in multiple countries $[11,23,24]$ during the pandemic. This rapid systematic review aims to outline current evidence regarding the associations of PA and exercise with depression and anxiety during the Covid-19 pandemic. 


\section{Methods}

In this rapid review, we sought for observational studies examining the associations of PA and depression and anxiety during the COVID-19 pandemic. Inclusion criteria were: (1) observational studies in any population, including cross-sectional and longitudinal designs. Longitudinal studies could be either prospective or retrospective; (2) studies have tested the association of PA with depression or anxiety, using linear or logistic regressions; (3) depression and anxiety were assessed using validated screening or diagnostic tools. We excluded opinion pieces, systematic reviews, and studies addressing other viruses.

We searched the electronic databases PubMed, EMBASE, SPORTDiscus, and Web of Science using the following strategy: (physical activity OR exercise OR sport) AND (coronavirus OR sars-cov-2 OR COVID* OR severe acute respiratory syndrome OR pandemic) AND (depression OR anxiety OR mental health). Preprints were searched in MedRxiv, SportRxiv, and SciELO Preprints using the following strategy: "(physical activity OR exercise) AND (coronavirus OR sars-cov-2 OR COVID* OR severe acute respiratory syndrome OR pandemic)". Additional hand searches were performed on COVID-19 online repositories on ResearchGate and Google Scholar. Searches were made by an experienced reviewer (FS) on 29th July, 2020. Study selection was conducted in three steps: (1) duplicates removal; (2) screening at the title and abstract level; and (3) assessment based on full-text. The selection was made by one reviewer (FS). Data extraction of selected studies was then performed by three researchers (FS, BS, SW). Data extracted were: author and year, country of the included sample, study design, sample size, age group of the sample included, when possible, mean or range of age sample, \% of women, instrument/question used to assess PA levels, instruments used to assess depression and anxiety, publication type and statistical outcomes (regression standardized beta coefficients and odd's ratios). If they were indicated in the report, fully adjusted coefficients and odd's ratios were extracted. As studies included in this review used very heterogeneous statistical approaches, a meta-analysis could not be conducted. Instead, we summarized the evidence and presented effect sizes [betas and odds ratios (OR)] with confidence intervals and indicated significant associations between PA and depression or anxiety, separately (see Table 2). In case the study just reported the unstandardized betas, we requested the standardized betas by e-mail. If standardized effects could not be obtained, unstandardized effects were presented and indicated. The risk of bias of individual studies was assessed using the National Institutes of Health (NIH) study quality assessment tool for observational cohort and cross-sectional studies [25]. The NIH tool assessment is composed by 14 questions the risk of potential selection bias, information bias measurement bias or confounding bias. There are three options (yes, no, other) for each question. Each "no" or "other" is suggestive of the presence of some risk of bias. Questions \#6 (exposure prior outcome), \#7 (sufficient time to see an effect), \#10 (repeated exposure assessment), and \#13 (follow-up rate) were disregarded for cross-sectional studies. Due to the self-reported nature of the assessments, question \#12 (blinding of outcome assessors) was also disregarded for all studies.

\section{Results}

Searches on PubMed, EMBASE, Sportdiscus, and Web of Science resulted in 592 potentially relevant studies. Preprint databases identified additional 572 potentially relevant studies. A flow-chart of the selection process is provided in Fig. 1. Of the identified studies, 21 studies meet the criteria [7, 8, 11, 26-43]. Four studies had a prospective longitudinal design [29, 32, 39, 42], 1 was a cross-sectional study with a retrospective measure of the exposure factor (henceforth treated as retrospective) [7], and 16 were cross-sectional studies [8, 11, 26-28, 30-38, 40, 41, 43, 44]. A total of 7 studies were conducted in Asia [27, 28, 30, 33, 36, 42, 43], 6 in Europe [11, 29, 34, 35, 38, 39], 3 in South America [8, 31, 32], 3 in North America [7, 26, 37], 1 in Oceania [41] and 1 study included a multinational sample [40].

Data form a total of 42,293 (median $=68 \%$ of women) participants were included. Only 1 study was exclusively composed by older adults (over 50 years), 4 were in children, adolescents, or young adults, while 13 studies were in adults (over 18). Only 7 studies used validated measures to assess PA levels. A wide range of scales to measure depression or anxiety were used, the most used scales being the Beck Depression and Anxiety inventory and the DASS-21. Most studies $(n=14)$ were peer-reviewed, seven studies were published as preprints. A summary of studies is provided in Table 1.

Results are summarized and presented in Table 2. Out of ten studies reporting analyses on the association between the overall volume of PA and depression, seven studies showed that more PA is significantly associated with less depression symptoms [26, 28, 30, 35, 36, 38, 40], and three out of nine studies investigating the association between the overall volume of PA and anxiety symptoms showed that more PA is significantly associated with less anxiety symptoms [28, $35,38]$. Three out of six studies reported higher frequencies of PA to be significantly associated with less depression [30, $32,39]$ and two out of five studies to be significantly associated with less anxiety [30, 32]. One study showed that vigorous but not moderate PA is significantly associated with 
Fig. 1 PRISMA flow-chart of the screening and selection of studies
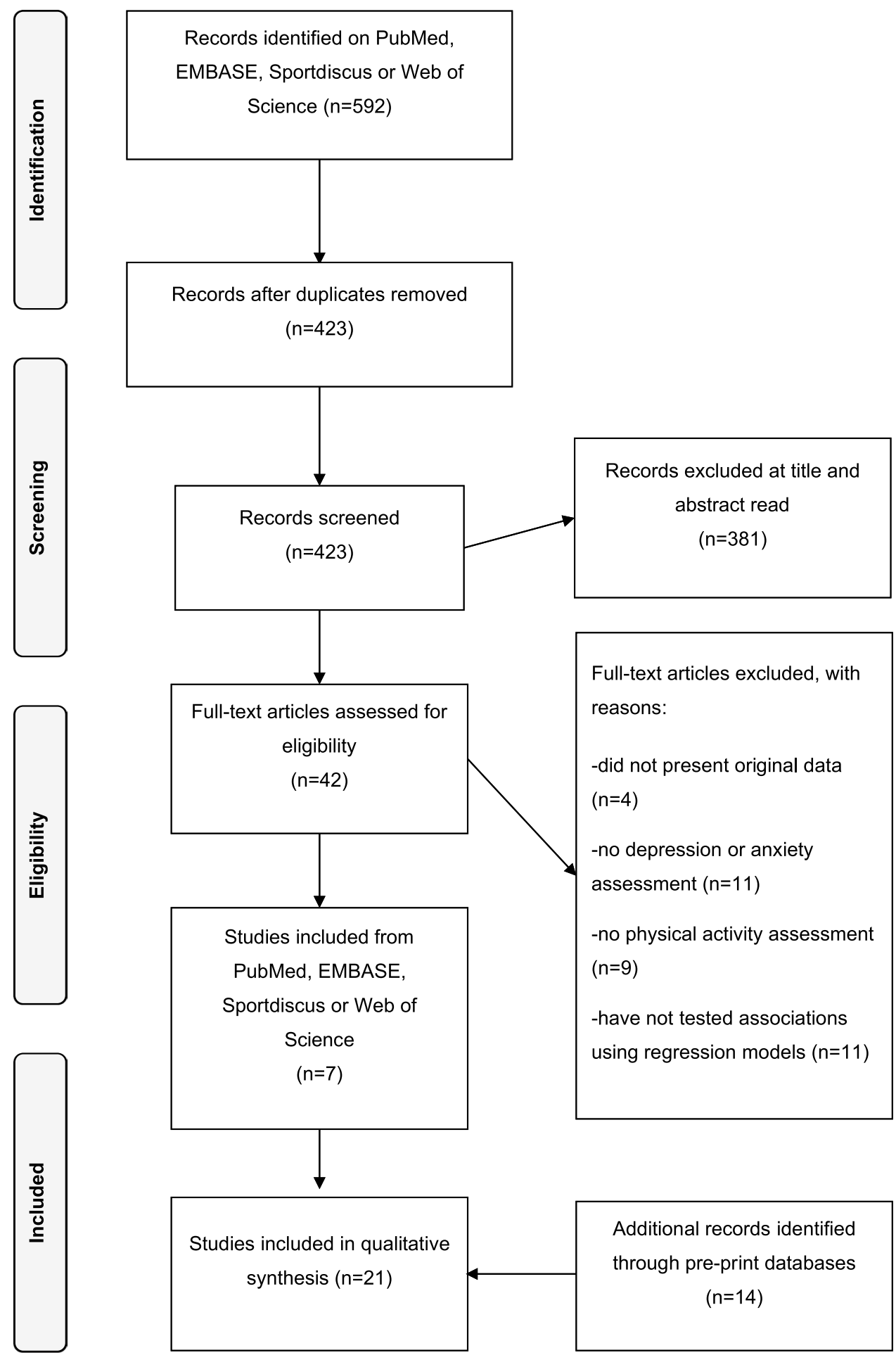

Records identified on PubMed,

EMBASE, Sportdiscus or Web of

Science $(n=592)$

Full-text articles excluded, with

reasons:

-did not present original data

$(n=4)$

-no depression or anxiety

assessment $(n=11)$

-no physical activity assessment

$(n=9)$

-have not tested associations

using regression models $(n=11)$

$(n=7)$

PubMed, EMBASE,

Sportdiscus or Web of

Science
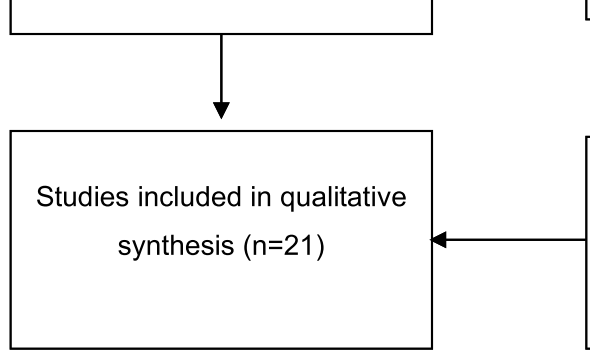

Additional records identified

through pre-print databases

$(n=14)$ less depression and anxiety symptoms [8] and another study indicated that light and vigorous PA is significantly correlated with less depression, but moderate intensity was not [26]. Out of four studies assessing an association between regular and guideline-consistent PA and depression and anxiety symptoms, two studies demonstrate that regular PA (compared to not regular) is significantly associated with less depression and anxiety symptoms [30, 33]. One study demonstrated that guideline conforming moderate to vigorous PA is associated with lower odds of depression and anxiety [8]. Five out of six studies showed that a decrease in PA during the pandemic was significantly associated with more depression symptoms [7, 11, 30, 41, 43] and three out of six studies showed that a decrease in PA was significantly 


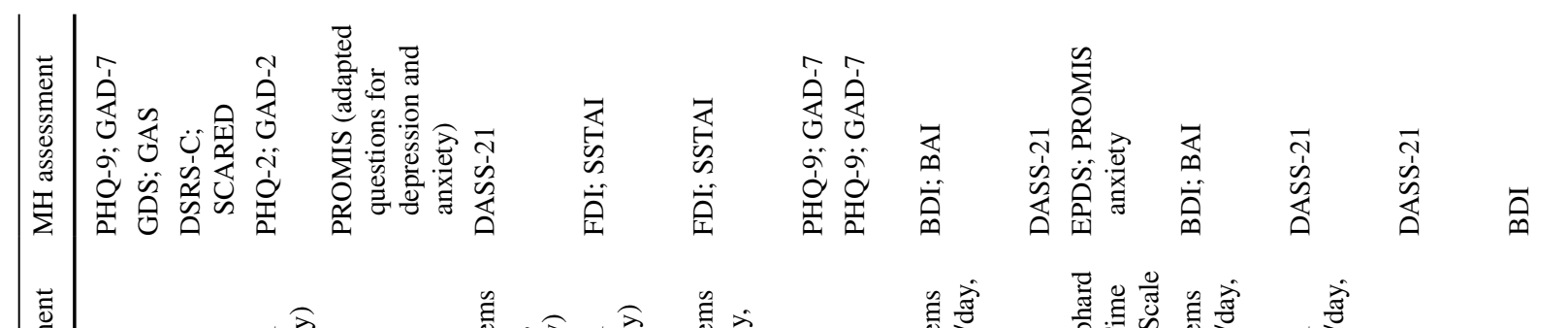

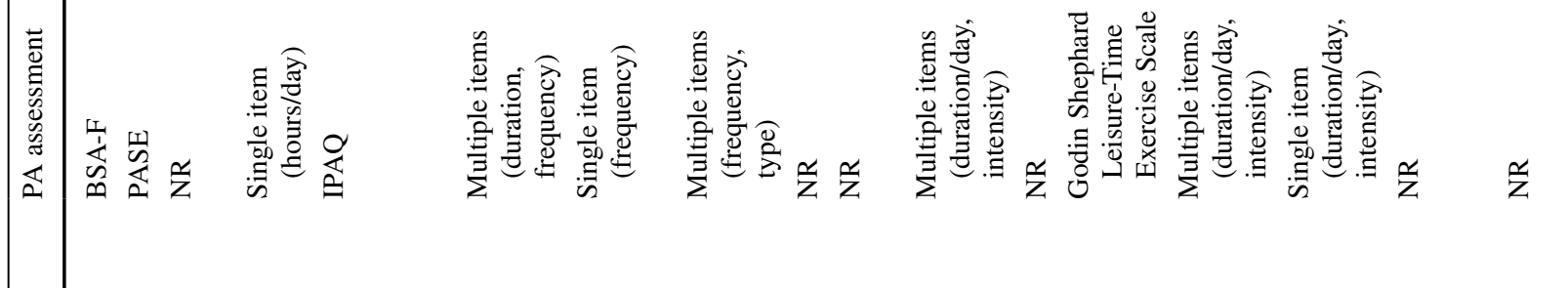

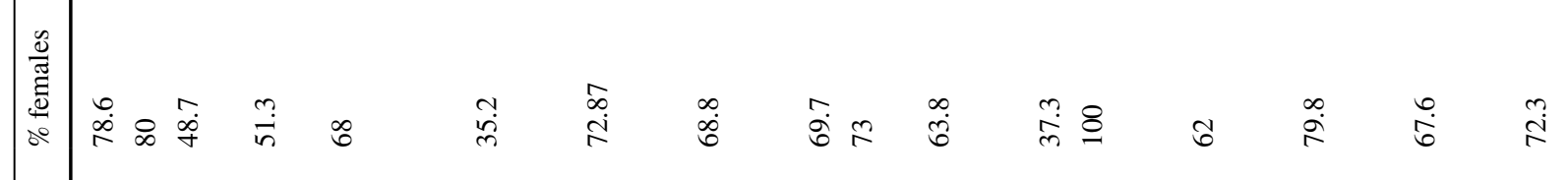

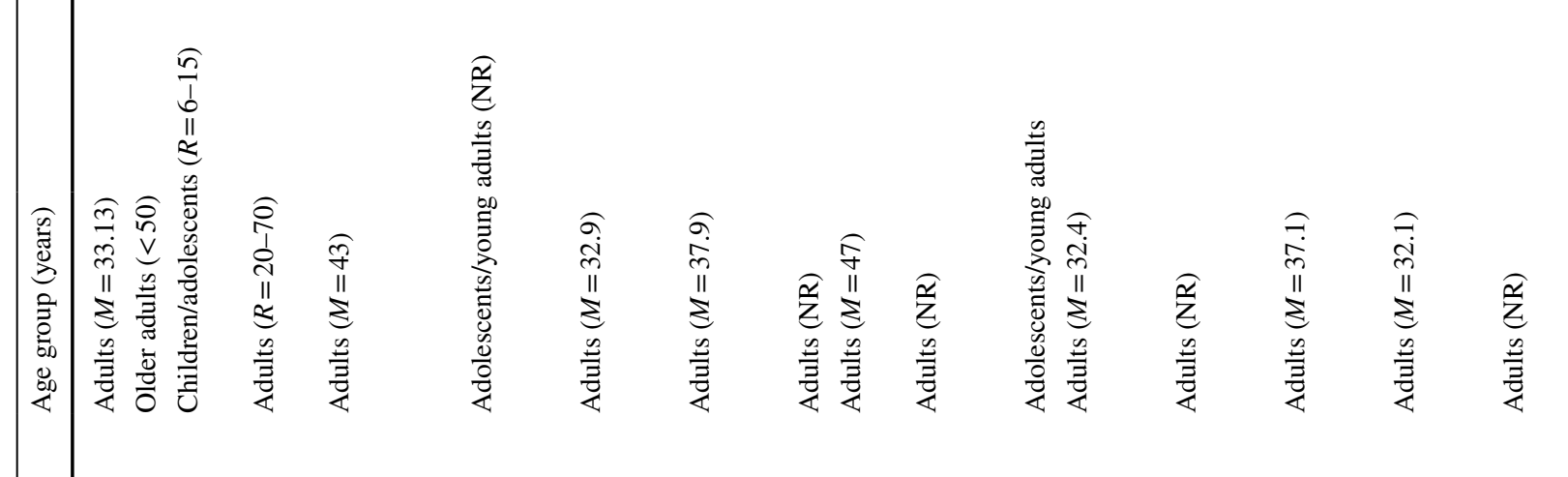

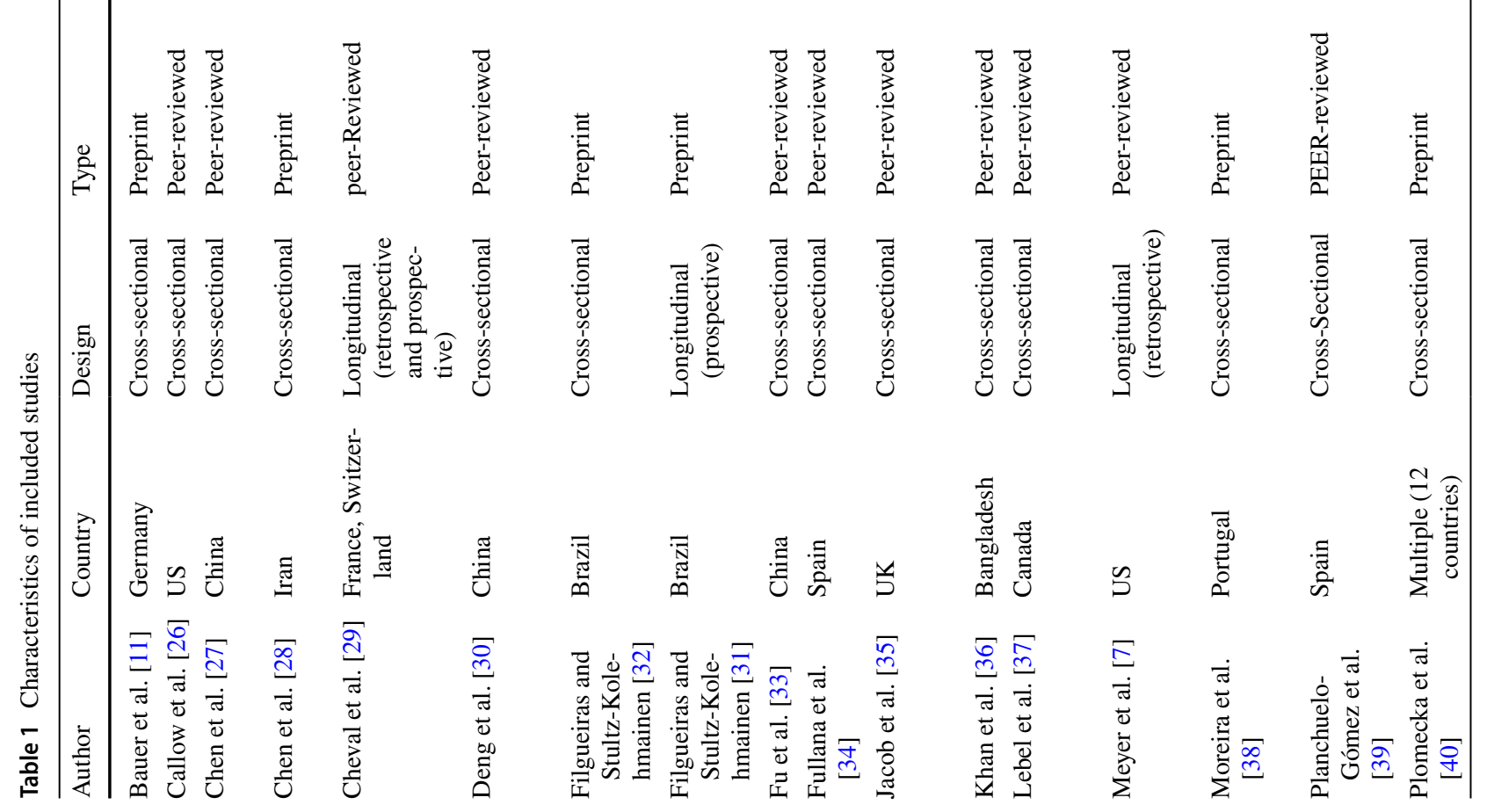

$$
\begin{aligned}
& \text { ๙ }
\end{aligned}
$$




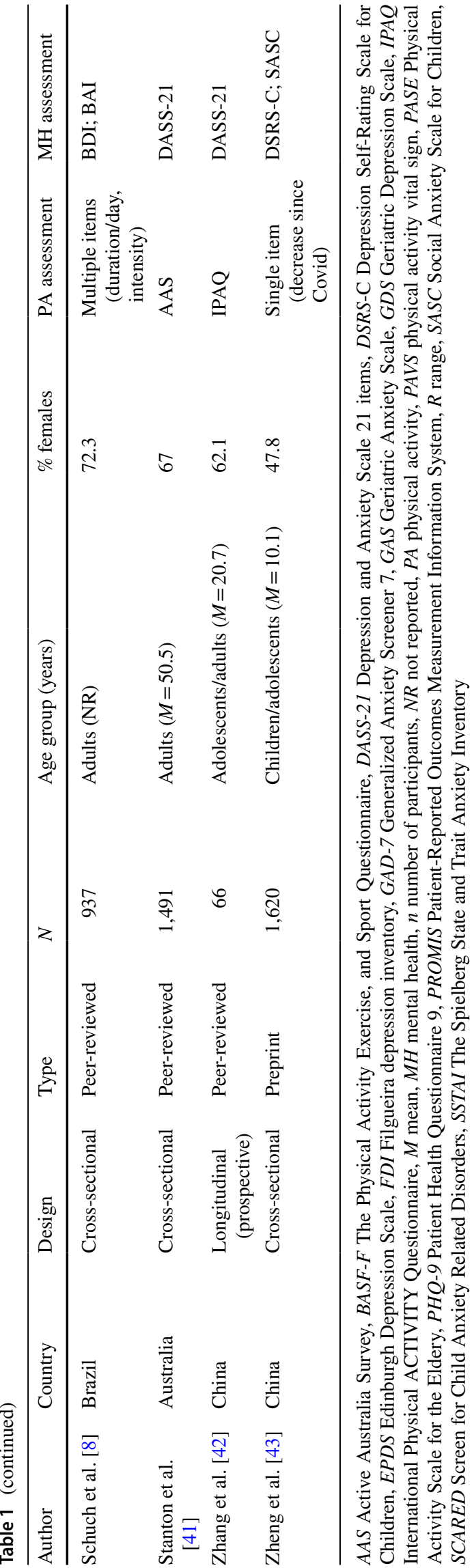

associated with more anxiety symptoms [11, 30, 41]. One study reported that an increase in PA was associated with less depressive symptoms [42].

The risk of bias of individual studies is presented in Table 3. All studies clearly defined their research questions and used valid tools to assess main outcomes. Among the cross-sectional studies, 11 (68.75\%) studies did not report the participation rate or included less than $50 \%$ of eligible participants, and $13(81.25 \%)$ did not use valid tools to assess the exposure measure. A total of three out of five $(60 \%)$ longitudinal studies are at risk of bias in the evaluating the definition of the study population, the participation rate, the validity of the exposure measure and in the retention of the sample.

\section{Discussion}

The present study is, to the best of our knowledge, the first study to summarize the evidence on the associations of PA with depression and anxiety during the COVID-19 pandemic. The majority of studies included in the present review showed that those who performed PA on a regular basis with higher volume and frequency and kept the PA routines stable, showed less symptoms of depression and anxiety. There was consistent evidence that those who could not keep their PA routine stable during the pandemic showed more depression and anxiety symptoms [7, 11, 30, 41-43]. However, the association was more consistent regarding depressive compared to anxiety symptoms. Those reporting a higher total time spent in moderate to vigorous PA had $12 \%$ to $32 \%$ lower chances of presenting depressive symptoms and $15-34 \%$ of presenting anxiety. These findings are in line with results of recent meta-analyses showing that those with higher PA levels were $17 \%$ less likely of developing depression [21] and 26\% less likely to develop anxiety [22] independently of the COVID-19 pandemic.

Indeed, the observed reduction in PA behavior during COVID-19 specific conditions is highly expected. For example, due to social distancing, exercising in a group setting was limited or completely prohibited. However, high social support is associated with more engagement in PA [45]. Indeed, social support was one of the strongest factors associated with adherence to PA in effective exercise interventions [46]. Furthermore, the COVID-19 pandemic impaired opportunities to be physically active due to the closure of sports clubs, gyms, or common indoor and outdoor places for PA. While some people were still allowed to do exercises like jogging on the streets, others were not [47]. In general, a lack of sporting opportunities seems to be associated with reduced PA [48]. Further negative consequences of the pandemic such as financial insecurities might have caused stress in individuals and 
Table 2 Main results of multiple linear and logistic regressions analyzing the association of physical activity or exercise with symptoms of depression or anxiety in the included studies

\begin{tabular}{|c|c|c|c|c|c|}
\hline \multirow[t]{2}{*}{ Author } & \multirow[t]{2}{*}{ Predictor } & \multicolumn{2}{|c|}{ Depressive symptoms } & \multicolumn{2}{|c|}{ Anxiety symptoms } \\
\hline & & Beta $(95 \% \mathrm{CI})$ & OR $(95 \% \mathrm{CI})$ & Beta $(95 \% \mathrm{CI})$ & OR $(95 \% \mathrm{CI})$ \\
\hline \multicolumn{6}{|l|}{ Volume } \\
\hline Bauer et al. [11] & $\begin{array}{l}\text { EX (minutes/ } \\
\text { week) }\end{array}$ & $\begin{array}{l}0.00 \#(\mathrm{NR} ; \\
\mathrm{NR})\end{array}$ & & $\begin{array}{l}0.01 \#(\mathrm{NR} ; \\
\mathrm{NR})\end{array}$ & \\
\hline Callow et al. [26] & PA (PASE score) & $\begin{array}{l}-0.22 * * * \\
(\mathrm{NR} ; \mathrm{NR})\end{array}$ & & $\begin{array}{l}-0.02(\mathrm{NR} ; \\
\mathrm{NR})\end{array}$ & \\
\hline Chen et al. [28] & EX (hours/day) & & $0.68 *(0.47 ; 0.97)$ & & $\begin{array}{l}0.66^{*}(0.45 \\
0.96)\end{array}$ \\
\hline Deng et al. [30] & $\begin{array}{l}\text { EX }(>60 \mathrm{~min} / \\
\text { day; } \\
\text { Ref: }<60 \mathrm{~min} / \\
\text { day }))\end{array}$ & $\begin{array}{c}-0.08 * * * \\
(\mathrm{NR} ; \mathrm{NR})\end{array}$ & & $\begin{array}{l}-0.05(\mathrm{NR} ; \\
\mathrm{NR})\end{array}$ & \\
\hline Cheval et al. [29] & PA (minutes/day) & $\mathrm{NR}^{\#}(\mathrm{NR} ; \mathrm{NR})$ & & $\mathrm{NR}^{\#}(\mathrm{NR} ; \mathrm{NR})$ & \\
\hline Jacob et al. [35] & $\begin{array}{l}\text { EX (minutes/ } \\
\text { day) }\end{array}$ & & $0.88^{\circ}(0.8 ; 0.97)$ & & $\begin{array}{l}0.85^{\circ}(0.79 \\
0.97)\end{array}$ \\
\hline Khan et al. [36] & $\begin{array}{l}\text { EX (any amount; } \\
\text { Ref.: No EX) }\end{array}$ & $\begin{array}{l}-2.1^{*}(-4.02 \\
-0.17)^{\mathrm{a}}\end{array}$ & & $\begin{array}{l}-0.55(-1.92 \\
0.82)^{\mathrm{a}}\end{array}$ & \\
\hline Moreira et al. [38] & EX (hours) & $\begin{array}{l}-1.17^{\circ}(\mathrm{NR} ; \\
\mathrm{NR})^{\mathrm{a}}\end{array}$ & & $\begin{array}{l}-0.81^{\circ}(\mathrm{NR} ; \\
\mathrm{NR})\end{array}$ & \\
\hline \multirow[t]{2}{*}{ Plomecka et al. [40] } & $\begin{array}{l}\text { EX }(>15 \mathrm{~min} / \\
\text { day; } \\
\text { Ref.: } \leq 15 \mathrm{~min} / \\
\text { day, }<60 \mathrm{~min} / \\
\text { day) }\end{array}$ & $\begin{array}{l}-0.13 * * * \\
(\mathrm{NR} ; \mathrm{NR})\end{array}$ & $\mathrm{NR}^{\#}(\mathrm{NR} ; \mathrm{NR})$ & & \\
\hline & $\begin{array}{l}\mathrm{EX}(\geq 60 \mathrm{~min} / \\
\text { day; } \\
\text { Ref.: } \leq 15 \mathrm{~min} / \\
\text { day) }\end{array}$ & $\begin{array}{c}-0.15 * * * \\
\quad(-0.18 \\
-0.12)\end{array}$ & $\mathrm{NR}^{\#}(\mathrm{NR} ; \mathrm{NR})$ & & \\
\hline Schuch et al. [8] & $\begin{array}{l}\text { PA (minutes/ } \\
\text { day; per } 10 \text { min } \\
\text { increase) }\end{array}$ & $\begin{array}{l}-0.03(-0.1 \\
0.03)\end{array}$ & & $\begin{array}{l}-0.05(-0.13 \\
0.02)\end{array}$ & \\
\hline \multicolumn{6}{|l|}{ Frequency } \\
\hline \multirow[t]{3}{*}{ Deng et al. [30] } & $\begin{array}{l}\text { EX }(1 \text { to } 2 \\
\text { times/week; } \\
\text { Ref: }<1 x / \text { week })\end{array}$ & $\begin{array}{l}-0.11 * * * \\
(\mathrm{NR} ; \mathrm{NR})\end{array}$ & & $\begin{array}{l}-0.09 * *(\mathrm{NR} \\
\mathrm{NR})\end{array}$ & \\
\hline & $\begin{array}{l}\text { EX }(>2 \\
\text { times/week; } \\
\text { Ref: }<1 x / \text { week })\end{array}$ & $\begin{array}{l}-0.15 * * * \\
(\mathrm{NR} ; \mathrm{NR})\end{array}$ & & $\begin{array}{l}-0.12 * *(\mathrm{NR} \\
\mathrm{NR})\end{array}$ & \\
\hline & $\begin{array}{l}\text { EX (every day; } \\
\text { Ref: <1x/week) }\end{array}$ & $\begin{array}{l}-0.11 * * * \\
(\mathrm{NR} ; \mathrm{NR})\end{array}$ & & $\begin{array}{l}-0.09 *(\mathrm{NR} \\
\mathrm{NR})\end{array}$ & \\
\hline Filgueiras and Stultz-Kolehmainen [32] & $\begin{array}{l}\text { EX (frequency/ } \\
\text { week) }\end{array}$ & $\begin{array}{l}-2.68^{* *}(\mathrm{NR} ; \\
\mathrm{NR})^{\mathrm{a}}\end{array}$ & & $\begin{array}{l}-1.64 * * * \\
(\mathrm{NR} ; \mathrm{NR})^{\mathrm{a}}\end{array}$ & \\
\hline Fullana et al. [34] & EX (Unclear) & & $0.93(\mathrm{NR} ; \mathrm{NR})$ & & $0.95(\mathrm{NR} ; \mathrm{NR})$ \\
\hline Lebel et al. [37] & $\begin{array}{l}\text { EX (Godin } \\
\text { Shephard } \\
\text { Leisure-Time } \\
\text { Exercise Score) }\end{array}$ & $\begin{array}{l}-0.01 *(\mathrm{NR} \\
\mathrm{NR})\end{array}$ & $0.99(0.99 ; 0.99)$ & $\begin{array}{l}-0.01 * *(\mathrm{NR} ; \\
\mathrm{NR})\end{array}$ & $0.99(0.99 ; 1.0)$ \\
\hline \multirow[t]{2}{*}{ Planchuelo-Gómez et al. [39] } & $\begin{array}{l}\text { EX (1-2 times/ } \\
\text { week; Ref.: No } \\
\text { EX) }\end{array}$ & $\begin{array}{l}-0.17(\mathrm{NR} ; \\
\mathrm{NR})^{\mathrm{a}}\end{array}$ & & & \\
\hline & $\begin{array}{l}\text { EX (3-5 times/ } \\
\text { week; Ref.: No } \\
\text { EX) }\end{array}$ & $\begin{array}{l}-0.85^{*}(\mathrm{NR} \\
\mathrm{NR})^{\mathrm{a}}\end{array}$ & & & \\
\hline
\end{tabular}


Table 2 (continued)

\begin{tabular}{|c|c|c|c|c|c|}
\hline \multirow[t]{2}{*}{ Author } & \multirow[t]{2}{*}{ Predictor } & \multicolumn{2}{|c|}{ Depressive symptoms } & \multicolumn{2}{|c|}{ Anxiety symptoms } \\
\hline & & Beta $(95 \% \mathrm{CI})$ & OR $(95 \% \mathrm{CI})$ & Beta $(95 \% \mathrm{CI})$ & OR $(95 \% \mathrm{CI})$ \\
\hline & $\begin{array}{l}\text { EX (6-7 times/ } \\
\text { week; Ref.: No } \\
\text { EX) }\end{array}$ & $\begin{array}{l}-1.29 * * * \\
(\mathrm{NR} ; \mathrm{NR})^{\mathrm{a}}\end{array}$ & & & \\
\hline $\begin{array}{l}\text { Filgueiras and Stultz-Kolehmainen } \\
\text { [31] }\end{array}$ & $\begin{array}{l}\text { EX (frequency/ } \\
\text { week) }\end{array}$ & $\mathrm{NR}^{\#}(\mathrm{NR} ; \mathrm{NR})$ & & $\mathrm{NR}^{\#}(\mathrm{NR} ; \mathrm{NR})$ & \\
\hline \multicolumn{6}{|l|}{ Intensity } \\
\hline \multirow[t]{3}{*}{ Callow et al. [26] } & $\begin{array}{l}\text { Light PA (PASE } \\
\text { score) }\end{array}$ & $\begin{array}{l}0.12 * *(\mathrm{NR} ; \\
\mathrm{NR})\end{array}$ & & & \\
\hline & $\begin{array}{l}\text { Moderate PA } \\
\text { (PASE score) }\end{array}$ & $\begin{array}{l}-0.01(\mathrm{NR} \\
\mathrm{NR})\end{array}$ & & & \\
\hline & $\begin{array}{l}\text { Vigorous PA } \\
\text { (PASE score) }\end{array}$ & $\begin{array}{l}0.09 *(\mathrm{NR} \\
\mathrm{NR})\end{array}$ & & & \\
\hline \multirow[t]{2}{*}{ Schuch et al., 2020 [8] } & $\begin{array}{l}\text { Vigorous PA } \\
\text { (minutes/day) }\end{array}$ & $\begin{array}{l}-0.19 * \\
\quad(-0.34 \\
-0.04)\end{array}$ & $0.6 * *(0.44 ; 0.83)$ & $\begin{array}{l}-0.22 *(-0.4 \\
-0.03)\end{array}$ & $\begin{array}{l}0.71 * *(0.52 \\
0.96)\end{array}$ \\
\hline & $\begin{array}{l}\text { Moderate PA } \\
\text { (minutes/day) }\end{array}$ & $\begin{array}{l}0.00(-0.09 \\
0.09)\end{array}$ & $0.77(0.57 ; 1.02)$ & $\begin{array}{l}-0.03(-0.14 \\
0.08)\end{array}$ & $0.75(0.58 ; 1)$ \\
\hline \multicolumn{6}{|l|}{ Regular/guideline conforming } \\
\hline Chen et al. [27] & $\begin{array}{l}\text { EX (regular; } \\
\text { Ref.: not regular) }\end{array}$ & & $0.37(\mathrm{NR} ; \mathrm{NR})^{\mathrm{b}}$ & & $0.43(\mathrm{NR} ; \mathrm{NR})^{\mathrm{b}}$ \\
\hline \multirow[t]{2}{*}{ Deng et al. [30] } & EX (regular; & $\begin{array}{l}-0.2 * * *(\mathrm{NR} \\
\mathrm{NR})\end{array}$ & & $\begin{array}{l}-0.14 * * * \\
(\mathrm{NR} ; \mathrm{NR})\end{array}$ & \\
\hline & Ref.: not regular) & & & & \\
\hline Fu et al. [33] & EX (not regular; & & $1.71 * * *(1.28 ; 2.29)$ & & $\begin{array}{l}1.45^{*}(1.08 \\
1.93)\end{array}$ \\
\hline Schuch et al. [8] & $\begin{array}{l}\text { PA }(\geq 30 \mathrm{~min} / \\
\text { day; } \\
\text { Ref.: }<30 \mathrm{~min} / \\
\text { day) }\end{array}$ & & $0.72 *(0.54 ; 0.96)$ & & $\begin{array}{l}0.72 *(0.54 \\
0.96)\end{array}$ \\
\hline \multicolumn{6}{|l|}{ Change } \\
\hline Bauer et al. [11] & $\begin{array}{l}\text { EX (less; equal; } \\
\text { more) }^{\mathrm{c}}\end{array}$ & $\begin{array}{l}-0.08 * * * \\
(\mathrm{NR} ; \mathrm{NR})\end{array}$ & & $\begin{array}{l}-0.05 * * * \\
(\mathrm{NR} ; \mathrm{NR})\end{array}$ & \\
\hline \multirow[t]{2}{*}{ Deng et al. [30] } & $\begin{array}{l}\text { EX (no change; } \\
\text { Ref.: large } \\
\text { change) }\end{array}$ & $\begin{array}{l}-0.27 * * * \\
(\mathrm{NR} ; \mathrm{NR})\end{array}$ & & $\begin{array}{l}-0.21 * * * \\
(\mathrm{NR} ; \mathrm{NR})\end{array}$ & \\
\hline & $\begin{array}{l}\text { EX (little change; } \\
\text { Ref.: large } \\
\text { change) }\end{array}$ & $\begin{array}{l}-0.22 * * * \\
(\mathrm{NR} ; \mathrm{NR})\end{array}$ & & $\begin{array}{l}-0.17 * * * \\
(\mathrm{NR} ; \mathrm{NR})\end{array}$ & \\
\hline Filgueiras et al. [31] & $\begin{array}{l}\text { EX (none, } \\
\text { increase, } \\
\text { decrease) }\end{array}$ & $\mathrm{NR}^{\#}(\mathrm{NR} ; \mathrm{NR})$ & & $\mathrm{NR}^{\#}(\mathrm{NR} ; \mathrm{NR})$ & \\
\hline \multirow[t]{3}{*}{ Meyer et al. [7] } & $\begin{array}{l}\text { PA (increased; } \\
\text { Ref.: main- } \\
\text { tained high) }\end{array}$ & $\begin{array}{l}-0.01(-0.05 \\
0.02)\end{array}$ & & $\begin{array}{l}0.00(-0.03 \\
0.04)\end{array}$ & \\
\hline & $\begin{array}{l}\text { PA (decreased; } \\
\text { Ref.: main- } \\
\text { tained high) }\end{array}$ & $\begin{array}{l}0.09 * * *(0.05 \\
0.13)\end{array}$ & & $\begin{array}{l}0.03(-0.01 \\
0.07)\end{array}$ & \\
\hline & $\begin{array}{l}\text { PA (maintained } \\
\text { low; Ref.: } \\
\text { maintained } \\
\text { high) }\end{array}$ & $\begin{array}{l}0.04(0.00 \\
0.07)\end{array}$ & & $\begin{array}{l}0.02(-0.02 \\
0.05)\end{array}$ & \\
\hline
\end{tabular}


Table 2 (continued)

\begin{tabular}{|c|c|c|c|c|c|}
\hline \multirow[t]{2}{*}{ Author } & \multirow[t]{2}{*}{ Predictor } & \multicolumn{2}{|c|}{ Depressive symptoms } & \multicolumn{2}{|c|}{ Anxiety symptoms } \\
\hline & & Beta $(95 \% \mathrm{CI})$ & OR $(95 \% \mathrm{CI})$ & Beta $(95 \%$ CI $)$ & OR $(95 \% \mathrm{CI})$ \\
\hline Stanton et al. [41] & $\begin{array}{l}\text { PA (negative } \\
\text { change; Ref.: } \\
\text { no change/posi- } \\
\text { tive change) }\end{array}$ & & $1.08 * * *(1.06 ; 1.11)$ & & $\begin{array}{l}1.09 * * *(1.05 ; \\
1.13)\end{array}$ \\
\hline Zhang et al. [42] & $\begin{array}{l}\text { PA (per } 100 \\
\text { MET increase) }\end{array}$ & $\begin{array}{l}-0.04 * \\
(-0.08 ; 0)\end{array}$ & & $\begin{array}{l}-0.03(-0.07 \\
0)\end{array}$ & \\
\hline Zheng et al. [43] & $\begin{array}{l}\text { PA (decrease } \\
\text { vs. no change/ } \\
\text { increase) }\end{array}$ & & $2.07 * *(\mathrm{NR} ; \mathrm{NR})$ & & $1.24(\mathrm{NR} ; \mathrm{NR})$ \\
\hline
\end{tabular}

$A O R$ adjusted odd's ratio, $E X$ exercise, $M E T$ metabolic equivalent of tasks, $N R$ not reported, $O R$ odd's ratio, $P A$ physical activity, $P A S E$ Physical activity Scale for the Elderly, Ref. reference category

${ }^{*} p<0.05 ; * *<<0.01 ; * * p<0.001 ;{ }^{\circ}$ significant association, $p$ value not reported; *no significant association, $p$ value not reported

${ }^{a}$ Unstandardized regression coefficient

${ }^{b}$ Odd's ratio calculated from case counts

${ }^{c}$ Post hoc analysis revealed that a decrease in exercise was significantly associated with less depression compared to stable exercise and increase. No other comparison reached significance

Table 3 Risk of bias assessment (NIHM tool for observational studies)

\begin{tabular}{|c|c|c|c|c|c|c|c|c|c|c|c|c|c|c|}
\hline Items & 1 & 2 & 3 & 4 & 5 & 6 & 7 & 8 & 9 & 10 & 11 & 12 & 13 & 14 \\
\hline \multicolumn{15}{|l|}{ Cross-sectional studies } \\
\hline Bauer et al. [11] & $\mathrm{Y}$ & $\mathrm{Y}$ & $\mathrm{Y}$ & $\mathrm{Y}$ & $\mathrm{Y}$ & - & - & $\mathrm{Y}$ & $\mathrm{Y}$ & - & $\mathrm{Y}$ & - & - & $\mathrm{Y}$ \\
\hline Callow et al. [26] & $\mathrm{Y}$ & $\mathrm{Y}$ & $\mathrm{Y}$ & $\mathrm{Y}$ & $\mathrm{Y}$ & - & - & $\mathrm{Y}$ & $\mathrm{Y}$ & - & $\mathrm{Y}$ & - & - & $\mathrm{Y}$ \\
\hline Chen et al. [27] & $\mathrm{Y}$ & $\mathrm{N}$ & NR & $\mathrm{N}$ & $\mathrm{Y}$ & - & - & $\mathrm{N}$ & $\mathrm{N}$ & - & $\mathrm{Y}$ & - & - & $\mathrm{N}$ \\
\hline Chen et al. [28] & $\mathrm{Y}$ & $\mathrm{Y}$ & $\mathrm{N}$ & $\mathrm{N}$ & $\mathrm{Y}$ & - & - & $\mathrm{Y}$ & $\mathrm{N}$ & - & $\mathrm{Y}$ & - & - & $\mathrm{Y}$ \\
\hline Deng et al. [30] & $\mathrm{Y}$ & $\mathrm{Y}$ & $\mathrm{Y}$ & $\mathrm{Y}$ & $\mathrm{N}$ & - & - & $\mathrm{Y}$ & $\mathrm{N}$ & - & $\mathrm{Y}$ & - & - & $\mathrm{N}$ \\
\hline $\begin{array}{l}\text { Filgueiras and Stultz-Kolehmainen } \\
\text { [32] }\end{array}$ & $\mathrm{Y}$ & $\mathrm{N}$ & NR & $\mathrm{Y}$ & $\mathrm{Y}$ & - & - & $\mathrm{Y}$ & $\mathrm{N}$ & - & $\mathrm{Y}$ & - & - & $\mathrm{Y}$ \\
\hline Fu et al. [33] & $\mathrm{Y}$ & $\mathrm{Y}$ & NR & $\mathrm{Y}$ & $\mathrm{Y}$ & - & - & $\mathrm{N}$ & $\mathrm{N}$ & - & $\mathrm{Y}$ & - & - & $\mathrm{Y}$ \\
\hline Fullana et al. [34] & $\mathrm{Y}$ & $\mathrm{Y}$ & NR & $\mathrm{Y}$ & $\mathrm{N}$ & - & - & $\mathrm{N}$ & $\mathrm{N}$ & - & $\mathrm{Y}$ & - & - & $\mathrm{Y}$ \\
\hline Jacob et al. [35] & $\mathrm{Y}$ & $\mathrm{Y}$ & NR & $\mathrm{Y}$ & $\mathrm{Y}$ & - & - & $\mathrm{Y}$ & $\mathrm{N}$ & - & $\mathrm{Y}$ & - & - & $\mathrm{Y}$ \\
\hline Khan et al. [36] & $\mathrm{Y}$ & $\mathrm{N}$ & NR & $\mathrm{Y}$ & $\mathrm{Y}$ & - & - & $\mathrm{N}$ & $\mathrm{N}$ & - & $\mathrm{Y}$ & - & - & $\mathrm{Y}$ \\
\hline Lebel et al. [37] & $\mathrm{Y}$ & $\mathrm{Y}$ & NR & $\mathrm{Y}$ & $\mathrm{Y}$ & - & - & $\mathrm{N}$ & $\mathrm{N}$ & - & $\mathrm{Y}$ & - & - & $\mathrm{Y}$ \\
\hline Moreira et al. [38] & $\mathrm{Y}$ & $\mathrm{Y}$ & NR & $\mathrm{Y}$ & $\mathrm{Y}$ & - & - & $\mathrm{N}$ & $\mathrm{N}$ & - & $\mathrm{Y}$ & - & - & $\mathrm{Y}$ \\
\hline Plomecka et al. [40] & $\mathrm{Y}$ & $\mathrm{Y}$ & $\mathrm{Y}$ & $\mathrm{Y}$ & $\mathrm{Y}$ & - & - & $\mathrm{Y}$ & $\mathrm{N}$ & & $\mathrm{Y}$ & & & $\mathrm{Y}$ \\
\hline Schuch et al. [8] & $\mathrm{Y}$ & $\mathrm{Y}$ & NR & $\mathrm{Y}$ & $\mathrm{Y}$ & & & $\mathrm{Y}$ & $\mathrm{N}$ & - & $\mathrm{Y}$ & - & - & $\mathrm{Y}$ \\
\hline Stanton et al. [41] & $\mathrm{Y}$ & $\mathrm{Y}$ & NR & $\mathrm{Y}$ & $\mathrm{Y}$ & - & - & $\mathrm{N}$ & $\mathrm{Y}$ & - & $\mathrm{Y}$ & - & - & $\mathrm{Y}$ \\
\hline Zheng et al. [43] & $\mathrm{Y}$ & $\mathrm{Y}$ & $\mathrm{Y}$ & $\mathrm{Y}$ & $\mathrm{N}$ & - & - & $\mathrm{N}$ & $\mathrm{N}$ & - & $\mathrm{Y}$ & - & - & $\mathrm{Y}$ \\
\hline \multicolumn{15}{|l|}{ Longitudinal studies } \\
\hline Cheval et al. [29] & $\mathrm{Y}$ & $\mathrm{N}$ & $\mathrm{Y}$ & $\mathrm{Y}$ & $\mathrm{Y}$ & $\mathrm{Y}$ & $\mathrm{Y}$ & $\mathrm{Y}$ & $\mathrm{Y}$ & $\mathrm{Y}$ & $\mathrm{Yt}$ & - & $\mathrm{Y}$ & NR \\
\hline $\begin{array}{l}\text { Filgueiras and } \\
\text { Stultz-Kolehmainen [31] }\end{array}$ & $\mathrm{Y}$ & $\mathrm{N}$ & NR & $\mathrm{Y}$ & $\mathrm{Y}$ & $\mathrm{Y}$ & $\mathrm{Y}$ & $\mathrm{Y}$ & $\mathrm{N}$ & $\mathrm{Y}$ & $\mathrm{Y}$ & - & $\mathrm{N}$ & $\mathrm{Y}$ \\
\hline Meyer et al. [7] & $\mathrm{Y}$ & $\mathrm{Y}$ & $\mathrm{Y}$ & $\mathrm{Y}$ & $\mathrm{Y}$ & $\mathrm{N}$ & $\mathrm{Y}$ & $\mathrm{Y}$ & $\mathrm{N}$ & $\mathrm{N}$ & $\mathrm{Y}$ & - & NA & $\mathrm{Y}$ \\
\hline Planchuelo-Gómez et al. [39] & $\mathrm{Y}$ & $\mathrm{Y}$ & NR & $\mathrm{Y}$ & $\mathrm{N}$ & $\mathrm{Y}$ & $\mathrm{Y}$ & $\mathrm{Y}$ & NR & $\mathrm{Y}$ & $\mathrm{Y}$ & - & $\mathrm{N}$ & $\mathrm{Y}$ \\
\hline Zhang et al. [42] & $\mathrm{Y}$ & $\mathrm{N}$ & NR & $\mathrm{Y}$ & $\mathrm{Y}$ & $\mathrm{Y}$ & $\mathrm{Y}$ & $\mathrm{Y}$ & $\mathrm{Y}$ & $\mathrm{Y}$ & $\mathrm{Y}$ & - & $\mathrm{Y}$ & NR \\
\hline
\end{tabular}

$Y$ yes, $N$ no, $N R$ not relevant stress, in turn, may differentially impact individuals' level of PA. Whereas habitually active individuals might even increase their level of PA, those who had not yet integrated exercise as a part of daily life, reduce their level of PA 
[49]. Thus, habitually active individuals might have built PA-related health competence and learned to utilize PA as a strategy to cope with negative feelings, such as stress, that may arise with sudden adaptions [50, 51]. Therefore, to prevent an increase in psychiatric disorders during the current or further pandemics, factors that facilitate the integration of PA into daily life routines, such as motivational and volitional skills, need to be identified and encouraged. Motivation and volition are core components of several theories of behavior change such as the Health Action Process Approach (HAPA) [52]. HAPA is a socialcognitive model specifying motivational and volitional determinants of health behavior such as building intentions for health behavior, planning the behavior, coping with specific challenges, maintaining the behavior, and perceiving self-efficacy for all processes. A recent metaanalysis shows that action self-efficacy has large effects on health behavior through intentions and maintenance self-efficacy [53]. Especially, self-efficacy in building intentions and action planning have larger effects on physical activity behavior compared with other health behavior [53]. Indeed, Covid-19 specific interventions should even more focus on self-efficacy experiences in building intentions for PA and performing PA, since PA routines are interrupted through anti-contagion policies. A widely used way to promote these motivational and volitional determinants is the application of behavior change techniques (BCTs) [54, 55]. During the COVID-19 pandemic, some BCTs appear to be particularly important for the maintenance of regular PA. For instance, the knowledge about the benefits of PA on symptoms that accompany lock-down procedures, such as lowered mood or anxiety might strengthen intentions for PA [4-6]. Furthermore, individuals need the strong ability of coping planning to anticipate barriers that could discourage them to engage in PA (e.g., curfew, closed facilities) and find strategies to overcome them (e.g., engage in home training).

A web-based tool, e.g., a smartphone application could be a low-threshold and cost-effective option to train, supervise, apply, and adopt such BCTs, especially in terms of COVID-19. First empirical evidence showed preliminary efficacy of apps in promoting PA. Users of such apps are more likely to meet recommendations on PA than non-users [56-59]. However, the evidence of long-term effects is currently inconclusive, since only few studies assess long-term effects. A current meta-analysis claims for more research to further elucidate the time course of intervention effects [59]. Furthermore, a meta-analysis showed that internet-delivered interventions, which are able to use different BCTs, were effective in increasing PA [60]. A major advantage of such web-based tools is the possibility to overcome some of the COVID-19 specific barriers. For instance, it is possible to become physically active online with friends or a virtual community, which might work against the lack of social support. In addition, limited sporting opportunities may be expanded through fitness technology and the provision of structured programs, as they can be used both indoors (e.g., through fitness videos) and outdoors (e.g., through running apps) and, therefore, be adapted to the specific situation.

\section{Limitations}

Most of the studies included in this review used cross-sectional research designs. A causal nature of these associations, therefore, remains unclear. There are notably differences in effect sizes which point at a high heterogeneity of the effects. Several studies further showed methodological shortcomings, e.g., not reporting the participation rate, including less than $50 \%$ of eligible participants, no validated tools to assess PA and failure to report standardized coefficients. Heterogeneity in research designs and statistical analyses hindered meta-analytic approaches, which would have provided a more sophisticated overall effect estimate. Finally, several included studies were published as preprints and are currently in review processes for final publications. It is, therefore, planned to update this review in the future.

\section{Conclusions, Future Research Directions, and Implications}

This rapid review shows promising evidence that higher volume and frequency of PA and the keeping of regular PA habits during the Covid-19 pandemic are associated with less symptoms of depression and anxiety. For instance, those reporting a higher total time spent in moderate to vigorous PA had 12-32\% lower chances of presenting depressive symptoms and $15-34 \%$ of presenting anxiety. Thus, the promotion of PA habits and routines might be a cost-effective and comprehensive worldwide applicable strategy to overcome the severe gap between people in need and people receiving mental health care, especially in low-income countries with even non-existing mental health supplies. Particularly, web-based technologies, could be an easily accessible way to increase motivation and volition for PA and maintain daily PA routines even under pandemic-specific barriers. However, only very few apps or websites have been tested in RCTs with high methodological standards [59, 61]. Thus, there is a clear need for more systematic research for effectively and safely usable apps or web-based programs to prevent psychiatric disorders through PA. 


\section{Declarations}

Funding Open Access funding enabled and organized by Projekt DEAL.

Conflict of interest All authors declare that they have no conflict of interest.

Availability of data and material Data sharing not applicable to this article since all data extracted from studies are presented in the current paper.

Author contributions SW and FS devised the project and the main conceptual ideas. FS performed the literature research and selection. FS, BS, and SW performed final data extraction. All authors significantly contributed to drafting and carefully reviewing the final manuscript.

Open Access This article is licensed under a Creative Commons Attribution 4.0 International License, which permits use, sharing, adaptation, distribution and reproduction in any medium or format, as long as you give appropriate credit to the original author(s) and the source, provide a link to the Creative Commons licence, and indicate if changes were made. The images or other third party material in this article are included in the article's Creative Commons licence, unless indicated otherwise in a credit line to the material. If material is not included in the article's Creative Commons licence and your intended use is not permitted by statutory regulation or exceeds the permitted use, you will need to obtain permission directly from the copyright holder. To view a copy of this licence, visit http://creativecommons.org/licenses/by/4.0/.

\section{References}

1. WHO. Overview of public health and social measures in the context of COVID-19. Interim Guidance. 2020 [cited 15.07.2020]. Available from: https://www.who.int/publications/i/item/ overview-of-public-health-and-social-measures-in-the-conte xt-of-covid-19.

2. Hsiang S, Allen D, Annan-Phan S, Bell K, Bolliger I, Chong $\mathrm{T}$, et al. The effect of large-scale anti-contagion policies on the COVID-19 pandemic. Nature. 2020;584(7820):262-7. https://doi. org/10.1038/s41586-020-2404-8.

3. Bendavid E, Oh C, Bhattacharya J, Ioannidis JPA. Assessing mandatory stay-at-home and business closure effects on the spread of COVID-19. Eur J Clin Invest. 2021. https://doi.org/10.1111/eci. 13484.

4. Brooks SK, Webster RK, Smith LE, Woodland L, Wessely S, Greenberg N, et al. The psychological impact of quarantine and how to reduce it: rapid review of the evidence. Lancet. 2020;395(10227):912-20. https://doi.org/10.1016/s01406736(20)30460-8

5. Lee AM, Wong JG, McAlonan GM, Cheung V, Cheung C, Sham PC, et al. Stress and psychological distress among SARS survivors 1 year after the outbreak. Can J Psychiatry. 2007;52(4):233-40. https://doi.org/10.1177/070674370705200405.

6. Yip PS, Cheung YT, Chau PH, Law YW. The impact of epidemic outbreak: the case of severe acute respiratory syndrome (SARS) and suicide among older adults in Hong Kong. Crisis. 2010;31(2):86-92. https://doi.org/10.1027/0227-5910/a000015.

7. Meyer J, McDowell C, Lansing J, Brower C, Smith L, Tully $\mathrm{M}$, et al. Changes in physical activity and sedentary behavior in response to COVID-19 and their associations with mental health in 3052 US adults. Int J Environ Res Public Health. 2020;17(18):6469. https://doi.org/10.3390/ijerph17186469.

8. Schuch FB, Bulzing RA, Meyer J, Vancampfort D, Firth J, Stubbs $\mathrm{B}$, et al. Associations of moderate to vigorous physical activity and sedentary behavior with depressive and anxiety symptoms in self-isolating people during the COVID-19 pandemic: a crosssectional survey in Brazil. Psychiatry Res. 2020;292:113339. https://doi.org/10.1016/j.psychres.2020.113339.

9. Cao W, Fang Z, Hou G, Han M, Xu X, Dong J, et al. The psychological impact of the COVID-19 epidemic on college students in China. Psychiatry Res. 2020;287:112934. https://doi.org/10. 1016/j.psychres.2020.112934.

10. Liu CH, Zhang E, Wong GTF, Hyun S, Hahm HC. Factors associated with depression, anxiety, and PTSD symptomatology during the COVID-19 pandemic: clinical implications for US young adult mental health. Psychiatry Res. 2020;290:113172. https://doi.org/ 10.1016/j.psychres.2020.113172.

11. Bauer LL, Seiffer B, Deinhart C, Atrott B, Sudeck G, Hautzinger $\mathrm{M}$, et al. Associations of exercise and social support with mental health during quarantine and social-distancing measures during the COVID-19 pandemic: a cross-sectional survey in Germany. medRxiv. 2020. https://doi.org/10.1101/2020.07.01.20144105.

12. Global Burden of Disease Collaborative Network. Global burden of disease study 2017 (GBD 2017) results. Seattle: Institute for Health Metrics and Evaluation (IHME); 2018.

13. Walker ER, McGee RE, Druss BG. Mortality in mental disorders and global disease burden implications: a systematic review and meta-analysis. JAMA Psychiat. 2015;72(4):334-41. https://doi.org/10.1001/jamapsychiatry.2014.2502.

14. Patel V. Global mental health: from science to action. Harv Rev Psychiatry. 2012;20(1):6-12. https://doi.org/10.3109/10673229. 2012.649108.

15. Thornicroft G, Chatterji S, Evans-Lacko S, Gruber M, Sampson N, Aguilar-Gaxiola S, et al. Undertreatment of people with major depressive disorder in 21 countries. Br J Psychiatry. 2017;210(2):119-24. https://doi.org/10.1192/bjp.bp.116. 188078.

16. WHO. Mental health ATLAS 2017. 2017 [cited 15.07.2020]. Available from: https://www.who.int/mental_health/evidence/ atlas/mental_health_atlas_2017/en/.

17. Moreno C, Wykes T, Galderisi S, Nordentoft M, Crossley N, Jones $\mathrm{N}$, et al. How mental health care should change as a consequence of the COVID-19 pandemic. Lancet Psychiatry. 2020;7(9):81324. https://doi.org/10.1016/S2215-0366(20)30307-2.

18. Holmes EA, O'Connor RC, Perry VH, Tracey I, Wessely S, Arseneault L, et al. Multidisciplinary research priorities for the COVID-19 pandemic: a call for action for mental health science. Lancet Psychiatry. 2020;7(6):547-60. https://doi.org/10.1016/ S2215-0366(20)30168-1.

19. Caspersen CJ, Powell KE, Christenson GM. Physical activity, exercise, and physical fitness: definitions and distinctions for health-related research. Public Health Rep. 1985;100(2):126-31.

20. WHO. Physical activity. 2018 [cited 15.07.2020]. Available from: https://www.who.int/news-room/fact-sheets/detail/physical-activ ity.

21. Schuch FB, Vancampfort D, Firth J, Rosenbaum S, Ward PB, Silva ES, et al. Physical activity and incident depression: a meta-analysis of prospective cohort studies. Am J Psychiatry. 2018;175(7):631-48. https://doi.org/10.1176/appi.ajp.2018.17111 194.

22. Schuch FB, Stubbs B, Meyer J, Heissel A, Zech P, Vancampfort $\mathrm{D}$, et al. Physical activity protects from incident anxiety: a meta-analysis of prospective cohort studies. Depress Anxiety. 2019;36(9):846-58. https://doi.org/10.1002/da.22915.

23. Schuch FB, Bulzing RA, Meyer J, López-Sánchez GF, Grabovac I, Willeit $\mathrm{P}$, et al. Moderate to vigorous physical activity and 
sedentary behavior change in self-isolating adults during the COVID-19 pandemic in Brazil: A cross-sectional survey exploring correlates. medRxiv. 2020. https://doi.org/10.1101/2020.07. 15.20154559.

24. Mattioli AV, Sciomer S, Cocchi C, Maffei S, Gallina S. Quarantine during COVID-19 outbreak: changes in diet and physical activity increase the risk of cardiovascular disease. NutrMetabCardiovasc Dis. 2020;30(9):1409-17. https://doi.org/10.1016/j.numecd.2020. 05.020 .

25. National Institutes of Health (NIH). Quality assessment tool for observational cohort and cross-sectional studies. 2014 [cited 15.07.2020]. Available from: https://www.nhlbi.nih.gov/healthpro/guidelines/in-develop/cardiovascular-risk-reduction/tools/ cohort.

26. Callow DD, Arnold-Nedimala NA, Jordan LS, Pena GS, Won J, Woodard JL, et al. The mental health benefits of physical activity in older adults: survive the COVID-19 pandemic. Am J Geriatr Psychiatry. 2020;28(10):1046-57. https://doi.org/10.1016/j.jagp. 2020.06.024.

27. Chen F, Zheng D, Liu J, Gong Y, Guan Z, Lou D. Depression and anxiety among adolescents during COVID-19: a cross-sectional study. Brain BehavImmun. 2020;88:36-8. https://doi.org/10. 1016/j.bbi.2020.05.061

28. Chen J, Zhang SX, Wang Y, Jahanshahi AA, Dinani MM, Madavani $A N$, et al. The curvilinear relationship between the age of adults and their mental health in Iran after its peak of COVID-19 cases. medRxiv. 2020. https://doi.org/10.1101/2020.06.11.20128 132.

29. Cheval B, Sivaramakrishnan H, Maltagliati S, Fessler L, Forestier C, Sarrazin P, et al. Relationships between changes in selfreported physical activity, sedentary behaviour and health during the coronavirus (COVID-19) pandemic in France and Switzerland. J Sports Sci. 2020;39:1-6. https://doi.org/10.1080/02640 414.2020.1841396.

30. Deng CH, Wang JQ, Zhu LM, Liu HW, Guo Y, Peng XH, et al. Association of web-based physical education with mental health of college students in Wuhan during the COVID-19 outbreak: cross-sectional survey study. J Med Int Res. 2020;22(10):e21301. https://doi.org/10.2196/21301.

31. Filgueiras A, Stults-Kolehmainen M. The relationship between behavioural and psychosocial factors among Brazilians in quarantine due to COVID-19. SSRN Electron J. 2020. https://doi.org/ $10.2139 /$ ssrn. 3566245 .

32. Filgueiras A, Stults-Kolehmainen M. Factors linked to changes in mental health outcomes among Brazilians in quarantine due to COVID-19. medRxiv. 2020. https://doi.org/10.1101/2020.05.12. 20099374.

33. Fu W, Wang C, Zou L, Guo Y, Lu Z, Yan S, et al. Psychological health, sleep quality, and coping styles to stress facing the COVID-19 in Wuhan, China. Transl Psychiatry. 2020;10(1):225. https://doi.org/10.1038/s41398-020-00913-3.

34. Fullana MA, Hidalgo-Mazzei D, Vieta E, Radua J. Coping behaviors associated with decreased anxiety and depressive symptoms during the COVID-19 pandemic and lockdown. J Affect Disord. 2020;275:80-1. https://doi.org/10.1016/j.jad.2020.06.027.

35. Jacob L, Tully MA, Barnett Y, Lopez-Sanchez GF, Butler L, Schuch F, et al. The relationship between physical activity and mental health in a sample of the UK public: a cross-sectional study during the implementation of COVID-19 social distancing measures. Ment Health Phys Act. 2020;19:100345. https://doi.org/ 10.1016/j.mhpa.2020.100345.

36. Khan AH, Sultana MS, Hossain S, Hasan MT, Ahmed HU, Sikder MT. The impact of COVID-19 pandemic on mental health and wellbeing among home-quarantined Bangladeshi students: a cross-sectional pilot study. J Affect Disord. 2020;277:121-8. https://doi.org/10.1016/j.jad.2020.07.135.
37. Lebel C, MacKinnon A, Bagshawe M, Tomfohr-Madsen L, Giesbrecht $\mathrm{G}$. Elevated depression and anxiety symptoms among pregnant individuals during the COVID-19 pandemic. J Affect Disord. 2020;277:5-13. https://doi.org/10.1016/j.jad.2020.07.126.

38. Moreira PS, Ferreira S, Couto B, Machado-Sousa M, Fernández M, Raposo-Lima C, et al. Protective elements of mental health status during the COVID-19 outbreak in the Portuguese population. medRxiv. 2020. https://doi.org/10.1101/2020.04.28.20080 671.

39. Planchuelo-Gomez A, Odriozola-Gonzalez P, Irurtia MJ, de LuisGarcia R. Longitudinal evaluation of the psychological impact of the COVID-19 crisis in Spain. J Affect Disord. 2020;277:842-9. https://doi.org/10.1016/j.jad.2020.09.018.

40. Plomecka MB, Gobbi S, Neckels R, Radziński P, Skórko B, Lazerri S, et al. Mental health impact of COVID-19: a global study of risk and resilience factors. medRxiv. 2020;12:983.

41. Stanton R, To QG, Khalesi S, Williams SL, Alley SJ, Thwaite TL, et al. Depression, anxiety and stress during COVID-19: associations with changes in physical activity, sleep, tobacco and alcohol use in Australian adults. Int J Environ Res Public Health. 2020;17(11):4065. https://doi.org/10.3390/ijerph17114065.

42. Zhang Y, Zhang H, Ma X, Di Q. Mental health problems during the COVID-19 pandemics and the mitigation effects of exercise: a longitudinal study of college students in China. Int J Environ Res Public Health. 2020;17(10):3722. https://doi.org/10.3390/ijerp h17103722.

43. Zheng Y, Li J, Zhang M, Jin B, Li X, Cao Z, et al. A survey of the psychological status of primary school students who were quarantined at home during the coronavirus disease 2019 epidemic in Hangzhou China. medRxiv. 2020. https://doi.org/10. 1101/2020.05.28.20115311.

44. Lopez-Bueno R, Calatayud J, Ezzatvar Y, Casajus JA, Smith L, Andersen LL, et al. Association between current physical activity and current perceived anxiety and mood in the initial phase of COVID-19 confinement. Front Psychiatry. 2020;11:729. https://doi.org/10.3389/fpsyt.2020.00729.

45. Bauman AE, Reis RS, Sallis JF, Wells JC, Loos RJF, Martin BW. Correlates of physical activity: why are some people physically active and others not? Lancet. 2012;380(9838):258-71. https://doi.org/10.1016/s0140-6736(12)60735-1.

46. Meade LB, Bearne LM, Sweeney LH, Alageel SH, Godfrey EL. Behaviour change techniques associated with adherence to prescribed exercise in patients with persistent musculoskeletal pain: systematic review. Br J Health Psychol. 2019;24(1):10-30. https://doi.org/10.1111/bjhp.12324.

47. Sallis JF, Adlakha D, Oyeyemi A, Salvo D. An international physical activity and public health research agenda to inform COVID-19 policies and practices. J Sport Health Sci. 2020;9(4):328-34. https://doi.org/10.1016/j.jshs.2020.05.005.

48. Burke SM, Carron AV, Eys MA. Physical activity context and university student's propensity to meet the guidelines Centers for Disease Control and Prevention/American College of Sports Medicine. Med SciMonit. 2005;11(4):CR171-6.

49. Stults-Kolehmainen MA, Sinha R. The effects of stress on physical activity and exercise. Sports Med. 2014;44(1):81-121. https://doi.org/10.1007/s40279-013-0090-5.

50. Buman MP, Tuccitto DE, Giacobbi PR. Predicting daily reports of leisure-time exercise from stress appraisals and coping using a multilevel modeling approach. J Sport Exercise Psychiatry. 2007;29:S150.

51. Sudeck G, Pfeifer K. Physical activity-related health competence as an integrative objective in exercise therapy and health sports-conception and validation of a short questionnaire. Ger J Exerc Sport Res. 2016;46(2):74-87. https://doi.org/10.1007/ s12662-016-0405-4. 
52. Schwarzer R. Modeling health behavior change: how to predict and modify the adoption and maintenance of health behaviors. ApplPsychol. 2008;57(1):1-29. https://doi.org/10.1111/j.14640597.2007.00325.x.

53. Zhang CQ, Zhang R, Schwarzer R, Hagger MS. A meta-analysis of the health action process approach. Health Psychol. 2019;38(7):623-37. https://doi.org/10.1037/hea0000728.

54. Abraham C, Kelly MP, West R, Michie S. The UK National Institute for Health and Clinical Excellence public health guidance on behaviour change: a brief introduction. Psychol Health Med. 2009;14(1):1-8. https://doi.org/10.1080/1354850080 2537903

55. Michie S, Ashford S, Sniehotta FF, Dombrowski SU, Bishop A, French DP. A refined taxonomy of behaviour change techniques to help people change their physical activity and healthy eating behaviours: the CALO-RE taxonomy. Psychol Health. 2011;26(11):1479-98. https://doi.org/10.1080/08870446.2010. 540664.

56. Coughlin SS, Whitehead M, Sheats JQ, Mastromonico J, Smith $\mathrm{S}$. A review of smartphone applications for promoting physical activity. Jacobs J Commun Med. 2016;2(1):021.
57. Carroll JK, Moorhead A, Bond R, LeBlanc WG, Petrella RJ, Fiscella K. Who uses mobile phone health apps and does use matter? A secondary data analytics approach. J Med Int Res. 2017;19(4):e125.

58. Gal R, May AM, van Overmeeren EJ, Simons M, Monninkhof EM. The effect of physical activity interventions comprising wearables and smartphone applications on physical activity: a systematic review and meta-analysis. Sports Med Open. 2018;4(1):42. https://doi.org/10.1186/s40798-018-0157-9.

59. Romeo A, Edney S, Plotnikoff R, Curtis R, Ryan J, Sanders I, et al. Can smartphone apps increase physical activity? Systematic review and meta-analysis. J Med Int Res. 2019;21(3):e12053. https://doi.org/10.2196/12053.

60. Davies CA, Spence JC, Vandelanotte C, Caperchione CM, Mummery WK. Meta-analysis of internet-delivered interventions to increase physical activity levels. Int J BehavNutrPhys Act. 2012;9(1):52. https://doi.org/10.1186/1479-5868-9-52.

61. Direito A, Dale LP, Shields E, Dobson R, Whittaker R, Maddison R. Do physical activity and dietary smartphone applications incorporate evidence-based behaviour change techniques? BMC Public Health. 2014;14(1):646. 